\title{
Location Based Crime Detection Using Data Mining
}

\author{
Dr. IshwarBaidari and S.P. Sajjan
}

\begin{abstract}
A huge amount of data set is generated every year on the basis of reporting of crime. This data can prove very useful in analyzing and predicting crime and help us to prevent the crime to some extent. Goal is to detect crime in a particular location using data mining techniques applied on mobile devices that were found during the occurrence of crime. Here we consider that each individual can be tracked by locating their mobile numbers and its digital traces. This approach finds usage not only in fighting against crime, but also effectively replace the current conventional methods, which in turn will save lots of time and money of the Government and other intelligent agencies. There had been an enormous increase in the crime in the recent years. ${ }^{[2]}$ The concern about national security has increased significantly since the 26/11 attacks at Mumbai, India. However, information and technology overload hinders the effective analysis of criminal and terrorist activities.
\end{abstract}

Keywords--- Crime Detection, Location Based Systems, Data Mining, Digital traces, Database, K Mean.

\section{INTRODUCTION}

$\mathrm{T}$ ODAY'S mobile handheld devices have become a feasible medium for carrying out an extensive variety of actions, involving information exchange. One of the important area insociety where mobile technology is gaining usage is crime prevention, crime detection and

reporting ${ }^{[5]}$. The high computational power of smart phones, tablets and PDAs accounts forhigh demand and usage by the general public. The development of cellular networkstechnologies is another main issue which makes mobile phone technology a viable mode forfighting crime.

Nowadays, more than $80 \%$ percent of population uses mobile phones. This form of telecommunication service is heavily dependent on the accurate determination of the handset locations to promptly switch from one service station to another. Telecommunication serversaccumulate huge amount of data that includes the recording of locations of handsets at certaintime intervals. Also, the phone numbers of both callers and called addressees are recorded. Suchdata is a tremendous resource of information that can be used for a wide variety of purposes,including, in particular, targeted advertisement, based on accumulated customer profiles.

Dr. IshwarBaidari, Currently Working as a Associate Professor in the Dept.of Computer Science.

S.P. Sajjan, Currently working as Teaching Assistant Professor(Contractual) in the Dept of Computer Scince, Central University, Karnatak.

DOI:10.9756/BIJSESC.8279
Currently, to the best of our knowledge, this kind of data is not used to detect suspiciousforms of behavior. A special data mining and reasoning technology is required toautomatically process the location data as its manual browsing is unaffordable. The lack ofadequate technology leads to disregarding a rich body of data of potential value in filteringout criminal behavior. Crimes might be prevented and networks of criminals groups withpeculiar inter-connections identified if it were possible to discover sets of unusual patterns ofcoordinated movement for groups of mobile phones.

\section{EXTRACTING THE BEHAVIOR PATTERNS}

To extract the behavioral patterns from the data that is derived from the formaldescription of human activity (in particular, the data on locations and calls), it is necessary tohave a computational model of participating (interacting) mobiles that simulates theirbehavior. We must reproduce the reasoning of wireless subscribers to hypothesize on theirmovements and calls to judge on the possibility of a criminal behavior for a selected group.

Having obtained the behavioral patterns as results of supervised machine learning, it ispossible to apply them to location and call data in real time. An algorithm is developed for aparticular location of about $100 \mathrm{~m}$ radius and various Classification, Clustering and Filteringmethods are applied.

\section{DATA Mining OVERVIEW}

We review the Crime Data Mining in two directions ${ }^{[2]}$

1) Crime Types and security concerns

2) Crime Data Mining Approaches used for crime prevention

\section{A. Crime types and Security Concerns}

Crime is defined as "an act or the commission of an act that is forbidden, or theomission of a duty that is commanded by a public law and that makes the offender liable topunishment by that law". An act of crime encompasses a wide range of activities, rangingfrom simple violation of civic duties (e.g., illegal parking) to internationally organized crimes (e.g., the 26/11 attacks).

The following are the different types of crimes

1) Property crime

2) Violent Crime

3) Crime against Women and Child

4) Traffic Violations

5) Terrorist's attacks and Other Offenses. 


\section{B. Data Used}

The recorded crime data is used for the study (based on mobile and other digital traces) as well as information collected during the investigation process.

The collected data set provides the following:

1) Location of Crime

2) Street address

3) Time of the crime

4) Type of Crime

5) Geographical information (x, y) coordinates within a range of radius "r" (say $100 \mathrm{~m}$ ).

\section{APPROACHES USED FOR CRIME PREVENTION}

\section{A. First Prevention Approach}

${ }^{[5]}$ Consider the case if someone is in danger and he or she is not able to call. He orshe may send a miss call to an emergency number which will activate and would send constant location to the dear ones and to the concerned nearest police station asking forhelp which may prove useful to track the person and prevent from any happening. The diagrammatic representation can be illustrated in Fig. 2 as follows:

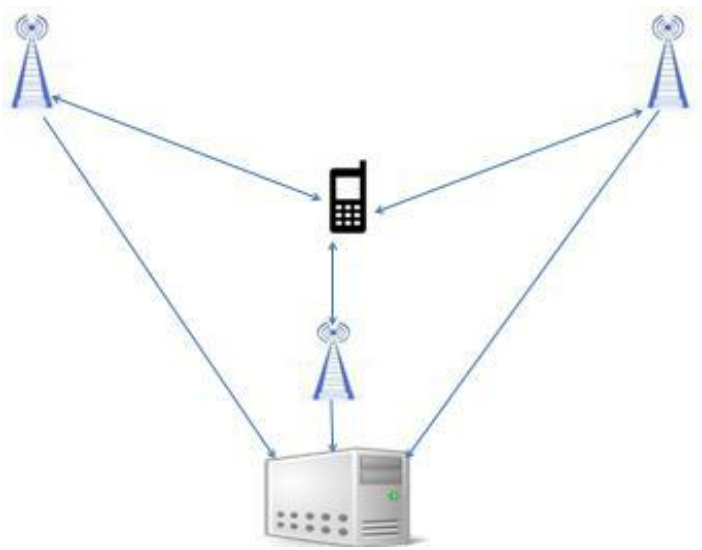

Above Fig1, contains:

1) Network Towers

2) Victim Mobile Phone

3) Server running to locate phone.

Fig. 1 depicts Pictorial representation to locate mobile upon getting a missed caller call, the algorithm will start running on a central server and it will send a locationrequest for the mobile phone. After receiving the location, the server will transfer custommessage to three registered numbers which is stored in the database respective to thecaller number with an SOS message along with the current location. The location will get updated after every 3 to 5 minutes and new message will be send. Moreover, theapplication can be enhanced with the feature of sending message to the nearest policestation or to the nearest police control room to take quick action. This will help the policeto prevent crime.

\section{B. Second Prevention Approach}

Suppose any criminal activity is reported then the reportcan be based on any of the following types:

1) If the criminal name is known, his or her current location can beobtained directly from the database together all other past records at the single click ofone command by proper administrator.

2) With the past records, criminals can be data mined and usingpredictive analysis; list of fewer criminals can be traced out from which the criminalcan be easily caught by direct investigations.

3) Again, a criminal is often motivated to do a similar kind of crimebased on research. So, it may prove useful to make use of this approach to list outfewer criminals which will help police to solve the crime.

\section{PROPOSED Methodology}

The main aim of this research work consist of developing analytical data miningmethods that can systematically address the complex problem related to various form ofcrime. The following illustrates the steps which can be followed to detect the Criminal:

1) Maintain a database of criminals. By collecting a log or historical Crime data ofminimum last 2 to 3 months and record the detail which forms our Dataset.

The Dataset must contain:

1) Locations of multiple mobiles

2) IMEI numbers of multiple mobiles

3) How often calls are made from multiple mobile numbers.

4) Common callers of multiple numbers

5) Maximum duration received \& call number

6) Minimum duration of Received \& call number

7) GPS Geo Position of Received \& called Number

8) All other parameters.

Below shows SQLqueryfor creating a new table of interest named Details from the huge database table named as CRIME_DB:

CREATE table Details as SELECT mobile, location, active, date FROM CRIME_DB;

2) Run Data mining Algorithms to fetch location of the mobile users and to filternew numbers with unusual behaviors which were recently active in that specificlocation among the existing old mobile numbers.

CREATE table New_Details as SELECT mobile, location, active, date FROM CRIME_DB GROUP By mobile HAVING location='Loc_name';

Various algorithms that can beused are:

a) Partition based algorithm (K Mean)

b) Density based algorithm

c) Hierarchical algorithm

d) Fuzzy C Mean algorithm

e) Expectation Maximization algorithm.

3) From list of different new mobiles (unusual behavior) obtained, will give us the reduced dataset with most probable criminal mobile number details.

Case1: The set of New or less frequently used mobile users amongst the Set of allusers who are continuous residents of particular locality. 


\section{SELECT * FROM Details INTERSECT SELECT * FROM New_Details;}

Case2: The set of mobiles which were found active during last few days duringthe Crime i.e. 2 to 3 days. The Set of mobiles recently found Active:

SELECT mobile, location from New_Details ORDER BY date Asc;

Case 3: Obtaining all mobile details that were intentionally switched off (disabled)after the Occurrence of Crime.

SELECT * FROM New_Details WHERE active='False' AND date $>26 / 8 / 16$;

Case4: Categorizing based on call durations:

a) Set of mobiles most frequently used: SELECT MAX(duration) FROM New_Details;

b) Set of mobiles less frequently used: SELECT MIN(duration) FROM New_Details;

4) Fetching a list of criminals in case criminals name is already registered for asimilar type crime and forwarding the list to the police to track the criminal.

${ }^{[3]}$ Following figure shows the methodology used for crime pattern analysis and detection:

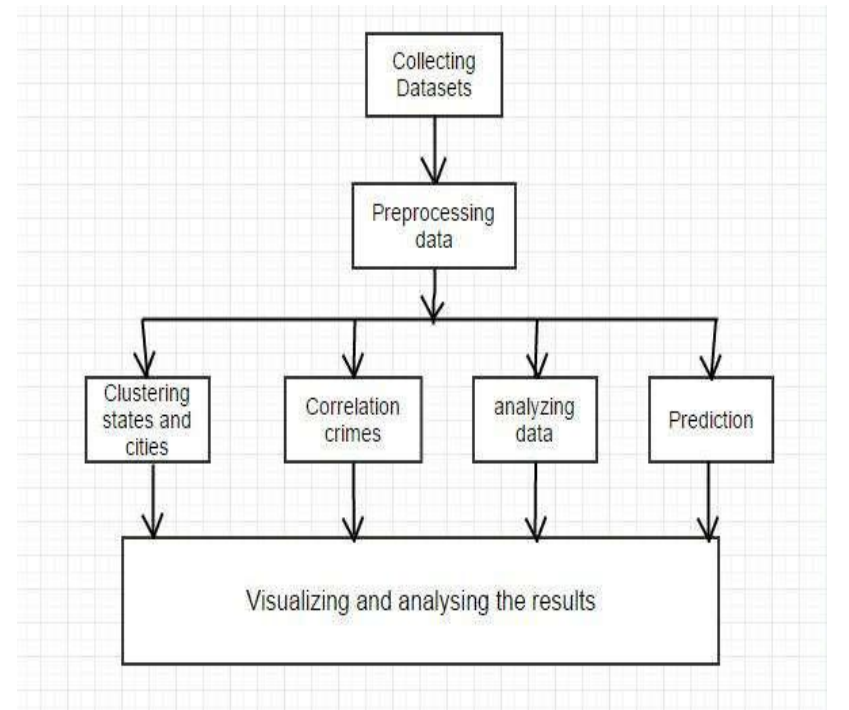

\section{A. Data Collection}

Enormous amount of crime data is collected at the end of year at police records. This data is made available by National Crime Bureau of Records. This data is in theform of number of cases recorded all over the nation throughout the year. The data is in raw form and also contains some wrong as well as missing values. Hence preprocessing of data becomes very necessary in order to bring the data in proper and clean form. Preprocessingof data includes data cleansing and PreProcessing.

\section{B. Data Classification}

We classify the data set into various groups based on certain characteristics of the data object here we group crimes according to states \& cities. Classification of the crime is done on the basis of different types of crime. $\mathrm{K}$ means algorithm can be used to group data with similar characteristics.

\section{Data Clustering}

a) Hard Clustering

These methods of clustering are based on the classical set theory, and require thatan object either does or does not belong to a cluster. It means partitioning the data intospecified number of mutually exclusive subsets. That is, each document belongs toexactly one cluster; it only assigns a value of 1 or 0 .

\section{b) K-Means Algorithm}

Mainly used to partition the clusters based on their means.Initially number of objects are grouped and specified as "K" clusters. The mean value iscalculated as the mean distance between the objects. The relocation iterative techniquewhich is used to improve the partitions by moving objects from one group to other. Thennumber of iterations is done until the convergence occurs. Steps are:

Input: Number of clusters.

Step 1: Arbitrarily choose $\mathrm{k}$ objects from a dataset $\mathrm{D}$ of $\mathrm{N}$ objects as the initial clustercenters.

Step 2: Reassign each object which distributed to a cluster based on a cluster centerwhich it is the most similar or the nearer.

Step 3: Update the cluster means, i.e. calculate the mean value of the object for eachcluster.

Output: A set of k clusters.

$\mathrm{K}$-means algorithm is a base for all other clustering algorithms to find the mean values.

c) Density-Based

This is based on connectivity and density functions. In density-based clustering, clusters are defined as areas of higher density than the remainder of the data set. Objectsin these sparse areas that are required to separate clusters - are usually considered to benoise and border points. The most popular density based clustering method is DBSCAN.In contrast to many newer methods, it features a well-defined cluster model called"density reach ability". Similar to linkage based clustering; it is based on connectingpoints within certain distance thresholds. However, it only connects points that satisfy adensity criterion, in the original variant defined as a minimum number of other objectswithin this radius.

\section{d) Hierarchical Algorithm}

Hierarchical clustering builds a cluster hierarchy (a tree of clusters). Create ahierarchical decomposition of the set of data (or objects) based on a criteria and usesdistance matrix as clustering criteria. This method does not require the number of clustersk as an input, but needs a termination condition. Connectivity based clustering, alsoknown as hierarchical clustering, is based on the core idea of objects being more relatedto nearby objects than to objects farther away. As such, these algorithms connect"objects" to form "clusters" based on their distance. A cluster can be described largely bythe maximum distance needed to connect parts of the cluster. At different distances, different clusters will be form, which can be represented using a dendrogram, whichexplains where the common name "hierarchical clustering".

\section{e) Fuzzy Clustering (Soft Clustering)}

Fuzzy clustering also called soft clustering. Allow the objects to belong to severalclusters simultaneously, with different degrees of membership. It is more natural thanhard 
clustering because objects on the boundaries between several classes are not forcedto fully belong to one of the classes, but rather are assigned membership degrees between 0 and 1 indicating their partial membership. For example, the clusters or groups that areidentified will be overlapping, meaning that one instance may fall into several clusters. Infuzzy clustering we make a fuzzy partition of the data set. This can be applied to crimeanalysis because police officers commonly receive descriptions of suspects that are fuzzy in nature. Fuzzy logic stipulates that an element can be a member of a given set in an uncertain manner. Unlike classical mathematical set theory in which an element can onlybe in two situations, a member or not a member of some set; fuzzy logic generalizes thepossibilities and introduces the concept of shades of membership patterns.

\section{f) Fuzzy C-Means}

Fuzzy C-Means Clustering (FCM) also known as Fuzzy ISODATA is a clusteringtechnique which is separated from hard k-means that employs hard partitioning. TheFCM employs fuzzy partitioning such that a data point can belong to all groups withdifferent membership grades between 0 and 1 . FCM is an iterative algorithm. The aim ofFCM is to find cluster centers (centroids) that minimize a dissimilarity function. Thisalgorithm works by assigning membership to each data point corresponding to eachcluster center on the basis of distance between the cluster center and the data point.

\section{g) Expectation Maximization Algorithm}

Expectation Maximization (EM) is a model based approach to solve clusteringproblem. It is an iterative algorithm that is used in problems where data is incomplete orconsidered incomplete. EM clusters data, in a manner different from K-means. It isknown to be an appropriate optimization algorithm for constructing proper statistical

Models of the data.

\section{Correlating Crime}

Many crimes are related to other crime or criminal. Finding this correlation can be of great help in finding missing clues. Correlations can be used to help make predictions. If two variables have been known in the past to correlate, then we can assume they will continue to correlate in the future. We can use the value of one variable that is known now to predict the value that the other variable will take on in the future .Pearson's correlation coefficient between two variables is defined as the covariance of the two variables divided by the product of their standard deviations.

\section{E. Predicting Crime}

Prediction of crime is a great aid to the administration in order to curb the crimeincidences. Prediction is stating the probability of occurrence of any similar crimes in future period time.

\section{RESULT}

The results of this data mining could potentially be used to lessen and even prevent crime for the forth coming years. From the encouraging results, we believe that crime data mining has a promising future for increasing the effectiveness and efficiencyof criminal and intelligence analysis. Many future directions can be explored in this stillyoung field. Visual and intuitive criminal and intelligence investigation techniques can bedeveloped for crime pattern

\section{APPLICATIONS}

The above defined approach is put in simpler format but it can be put to use intracking many different things. Firstly to predict the crimes from huge data set available.Secondly, it can replace the exhaustive GPS technology for monitoring the location ofvehicles by transport agencies. The approach would prove to be cost effective since itdoes not require much energy in transmitting large bandwidth signals as in the case ofGPS technology and since less bandwidth is used so lesser traffic and hence cheapermeans of locating vehicles. Also, as already mentioned, it can be put to use by the policedepartment to keep track of criminals and by using predictive analysis approach, providea list of criminals to the police to make their hard work easy in a sense to crack the case.Moreover, it can help to prevent from many future crimes to happen since it can help thepolice to track the victim.

\section{CONCLUSION AND FUTURE ENHANCEMENT}

This suggested a new way of extracting criminal behavior, mining the data oflocation-based services. The aim is to provide with the setting up of infrastructure andbasic approach to help prevent crime using information technology. We presented thetypes of crime and how it can be handled by data mining tool. By using this it is veryuseful to detect crime patterns and analyze crime analysis. It reduce further occurrence ofsimilar incidence. It formulates strategies for crime prevention and reduction. Moreover,other branches of study like usage of predictive analysis, artificial intelligence, and big dataapproach on large data sets can be put together to enhance the crime prevention system.Also, above approach can be put in different applications like vehicle location detection intransport industries.

\section{REFERENCES}

[1] D.E. Brown, "The regional crime analysis program (RECAP): A frame work formining data to catch criminals", In International Conferenceon Systems Proceedings, Man, and Cybernetics, Vol. 3, Pp. 2848-2853, 1998.

[2] A. Malathi, S.S. Babboo and A. Anbarasi, "An intelligent analysis of a city crime data using data mining", In International conference information electronic engineering, Vol. 6, Pp. 130-134, 2011.

[3] H. Chen, W. Chung, J.J. Xu, G. Wang, Y. Qin and M. Chau, "Crime data mining: a general framework and some examples", Computer, Vol. 37, No. 4, Pp. 50-56, 2004.

[4] TextBook entitled, "Data Mining for Intelligence, Fraud, Detection Advance Analytics andinformation sharing", by Christopher Westphal.

[5] S. Syal and Dr.M. Singh, "Prevent Crime Using Mobile- A Practical Approach", Vol.4, 2014.

[6] B. Prem Kumar and M. Stanislas Ashok, "A Survey of Positioning Algorithms onMobile Devices in Location Based Services", International Journal of AdvancedResearch in Computer Science and Software Engineering, Vol. 3, No. 6, 2013.

[7] B. Chandra and M.P. Gupta, "Crime Data Mining for Indian Police InformationSystem Manish Gupta”, Indian Institute of Technology.

[8] R. Obi Okonkwo "Combating Crime and Terrorism Using Data Mining Techniques”, ITePED 2011. 
[9] L. McClendon and N. Meghanathan, "Using Machine Learning Algorithms to Analyze Crime Data", Machine Learning and Applications: An International Journal (MLAIJ), Vol, 2.

[10] J.A. Adeyiga and A.O. Bello, "A Review of Different Clustering Techniques in Criminal Profiling", Vol. 6, No. 4, 2016.

[11] M. Ramzan Begam, "Survey: Tools and Techniques implemented in Crime Data Sets", IJISET, Vol. 2, No. 6, 2015.

[12] R. Vignesh Kumar, "Mobile Theft Detection With Automatic Location Tracking", IJAE, 2015.

[13] Malathi, "An Enhanced Algorithm to Predict a Future Crime Using Data Mining", Vol. 21, 2011.

[14] Sukanya, "Criminals and Crime Hotspot Detection Using Data Mining algorithms: Clustering and Classification",.

[15] Vineet Pande, "Crime Detection Using Data Mining", IJERT, Vol. 5, 2016.

[16] P.S. Gaurav Kumar, "Smart Security by Predicting Future Crime with GIS and LBS Technology on Mobile", Vol. 5, No. 2, 2016.

[17] S. Khan, "Data Mining For Security Purposes and its Solitude Suggestions", IJSTR, Vol. 1, 2012.

\section{AUTHORS PROFILE}

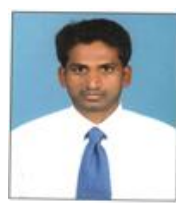

Dr. IshwarBaidari, pursed Bachelor of Science from Karnatak University, Dharwad and awarded Ph.D degree from the same University,currentlyworking as a Associate Professor in the Dept.of computer Science. His main research work focuses on Graph Algorithms and Big data Analytics. He has more than 10 years of experience.

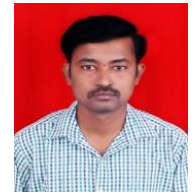

S.P. Sajjan, Currently working as teaching assistant professor(Contractual) in the Dept of Computer Scince , Central University, Karnatak. Mr. S P Sajjan obtain his post gradution(M.Sc) from Karnatak University, Dharwad, He has Four year Experience in Teaching and Industry, He has worked three years as a Teachig assistant in Karnatak University, Dharwad. 